\title{
ZERO DIVISORS AND FINITE NEAR-RINGS
}

S. LIGH and J. J. MALONE, Jr.

(Received 25 March 1969, revised 6 June 1969)

Communicated by G. B. Preston

A near-ring is a triple $(R,+, \cdot)$ such that $(R,+)$ is a group, $(R, \cdot)$ is a semigroup, and is left distributive over + ; i.e. $w(x+z)=w x+w z$ for each $w, x$, $z \in R$. The most comprehensive work on near-rings is [1]. A near-ring $R$ is distributively generated if there exists $S \subset R$ such that $(S, \cdot)$ is a sub-semigroup of $(R, \cdot)$, each element of $S$ is right distributive, and $S$ is an additive generating set for $(R,+)$. Distributively generated near-rings, first treated in [3], arise out of consideration of the system generated by the endomorphisms of an (not necessarily commutative) additive group. A near-field is a near-ring such that the nonzero elements form a group under multiplication. Near fields are discussed in [9]. An element $x \neq 0$ in $R$ is a left (right) zero divisor if there is $a \neq 0$ in $R$ such that $x a=0(a x=0)$. A zero divisor is an element that is either a left or a right zero divisor. In a near-ring $R$ it will be assumed that $0 x=0$ for each $x \in R$.

In Section 1 near-rings with no zero divisors are studied. In Section 2 it is shown that a near-ring with a finite number of zero divisors is finite. This generalizes a ring theoretic result. In Section 3 a ring theoretic result concerning elements that are not zero divisors is generalized to distributively generated near-rings.

\section{Near-rings with no zero divisors}

In this section it is assumed that all near-rings dealt with are finite and have no zero divisors.

Lemma 1.1. Let $R$ be a near-ring. For each nonzero $x \in R$ there exists a least positive integer $n$ such that $x^{n+1}=x$ and, for this $n, x^{n}$ is a left identity. In particular, if $x^{2}=x$ then $x$ is a left identity.

Proof. Since $\left\{x^{n} \mid n\right.$ a positive integer $\}$ is finite, it follows that for each nonzero $x$ there exists a least positive integer $n$ such that $x^{n} x=x$. As in the case of rings without zero divisors, one proves, by using left distributivity, that $R=x R$. But $R=x R$ and $x^{n} x=x$ imply that $x^{n}$ is a left identity. In particular, if $n=1$ then $x$ is a left identity.

THEOREM 1.2. If $R$ has a nonzero right distributive element, then $R$ is a nearfield and $(R,+)$ is a commutative group. 
Proof. Let $x \in R, x \neq 0$, be right distributive. By Lemma 1.1 there exists a positive integer $n$ such that $x^{n}$ is a left identity. From $\left(w x^{n}-w\right) x=0, w \in R$, it follows that $x^{n}$ is also a right identity. For an arbitrary nonzero $w \in R, R=w R$ so that there exists $z \in R$ such that $x^{n}=w z$. Thus $(R-\{0\}, \cdot)$ is a group. It was proved in [8] that the additive group of a near-field is commutative. (H. Zassenhaus [10] had previously shown that the additive group of a finite near-field is commutative.)

COROLLARY 1.3. If $R$ has a unique left identity, then $R$ is a near-field.

Proof. Let $e$ be the unique left identity. For nonzero $x \in R, x^{n} x=x=x x^{n}$ and $x^{n} y$ for each $y \in R$. Thus $e=x^{n}$ and $x=x e$ so that $e$ is a right identity. But, as a right identity, $e$ is right distributive.

LeMMA 1.4. $[5$, p. 60] Let $(G,+)$ be a finite group with an automorphism $\alpha$ such that $\alpha^{2}=I$ and such that 0 is the only fixed point for $\alpha$. Then $G$ is commutative.

THEOREM 1.5. Let $R$ be a near-ring such that $(R,+)$ is noncommutative. Then for each $x \in R$ there is a unique $y \in R$ such that $x=y^{2}$.

Proof. Let $x \in R, x \neq 0$, and let $n$ be the positive integer of Lemma 1.1. Assume $n=2 k$, where $k \geqq 1$. Consider the map $\alpha:(R,+) \rightarrow(R,+)$ defined by $(y) \alpha=x^{k} y$. It is immediate that $\alpha$ is an automorphism and that $\alpha^{2}=I$. Suppose there exists a nonzero $y \in R$ such that $x^{k} y=y$. Since $y R=R$ there exists $y^{\prime} \in R$ such that $y y^{\prime}=x^{2 k}$. Then $x^{k} y y^{\prime}=y y^{\prime}=x^{2 k}$, and $x^{k}=x^{2 k}$. From this contradiction it follows that such a $y$ does not exist. Thus $\alpha$ satisfies the conditions of Lemma 1.4 and $(R,+)$ is commutative. This contradiction implies that $n$ is odd. Thus $n+1$ is even, say $n+1=2 m$, and $\left(x^{m}\right)^{2}=x$. Also if $\left(x^{t}\right)^{2}=x$, then $t \geqq m$.

It remains to be shown that if $y^{2}=x$, then $y=x^{m}$. From the first part of the proof, there exists a least positive integer $t$ such that $\left(y^{t}\right)^{2}=y$. Since $y^{2}=x$, it follows that $y=y^{2 t}=x^{x}$. This implies that the order of $y$ (in the multiplicative group generated by $x$ ), namely $2 t-1$, divides $2 m-1$. Thus $t \leqq m$, so that $t=m$, and $y=x^{m}$.

EXAMPLE 1.6. The near-ring on $\left(Z_{5},+\right)$ gives as $\# 7$ in [2, Section 2.3] shows that Theorem 1.5 cannot be extended to near-rings defined on commutative groups.

On the elements of any group $(G,+)$ the multiplication defined by $0 g=0$ and $g_{1} g=g$ for $g_{1} \neq 0$ and $g \in G$ is such that $(G,+, \cdot)$ is a near-ring. This is one of the "trivial" multiplications discussed in [7]. For several classes of groups, this is the only near-ring with no zero divisors definable on the groups. Some such classes are given below.

Let $R$ be a near-ring and let $x \in R, x \neq 0$. The map $\alpha_{x}: R \rightarrow R$ defined by $(y) \alpha_{x}=x y$ is an automorphism of $(R,+)$. Thus each row in the multiplication table of $R$ may be considered to be (the images under) an automorphism. If $x$ is 
such that there exists a nonzero $y \in R$ so that $x y=y$, then $x z=z$ for each $z \in R$. This follows since $R=y R$ and $z$ may be written as $y w$ for some $w \in R$. This leads to

THEOREM 1.7. If $(R,+)$ is a complete group, then the near ring $R$ has the trivial multiplication.

Proof. Since the inner automorphism determined by conjugation by a nonzero $x$ leaves $x$ fixed, this result follows from the discussion above.

It is clear that in order for $R$ to have a non-trivial multiplication, $(R,+)$ must have at least one fixed point free automorphism. The dihedral group $D_{8}$ has no fixed point free automorphism and so admits only the trivial multiplication. Also, a group with a unique element of order 2 such as the quaternion group $Q_{8}$ would admit only the trivial multiplication.

Again, for $x \neq 0$, in $R$, let $n$ be the positive integer of Lemma 1.1. Then for $1 \leqq t<k \leqq n$, there cannot be a nonzero $y$ such that $x^{k} y=x^{t} y$. For if $k=t+c$, it would follow that $x^{c} y=y$, with $c \leqq n-1$. From this contradiction it is seen that $x y, x^{2} y, \cdots, x^{n} y=y$ are distinct. Recalling that the $\alpha_{x} i, i=1, \cdots, n$, where $(y) \alpha_{x} i=x^{i} y$, are automorphisms, one sees that the elements $(y) \alpha_{x} i$, $i=1, \cdots, n$, are distinct elements of order $|y|$. Therefore $n$ is less than or equal to the number of elements of order $|y|$ for each nonzero $y \in R$. Of course, $n$ is the order (in the group of automorphisms of $(R,+)$ ) of the automorphism associated with left multiplication by $x$.

\section{Near-rings with a finite number of zero divisors}

In this section the zero element is also taken to be a zero divisor. K. Koh [6] has shown that a ring having $n+1$ left (right) zero divisors, $n$ a positive integer, is finite and does not contain more than $(n+1)^{2}$ elements. In this section Koh's result is extended to near-rings.

THEOREM 2.1. Let $R$ be a near-ring with $n+1$ right zero divisors. Then $R$ is finite and does not contain more than $(n+1)^{2}$ elements.

Proof. For each $y \in R$, define $R_{y}=\{x \in R \mid y x=0\}$. Clearly $R_{y}$ is a subgroup of $R$. Since $R$ has $n+1$ right zero divisors, there is $a \in R$ such that $R_{a} \neq 0$ and the order of $R_{a}$ is at most $n+1$. For otherwise $R$ has more than $n+1$ right zero divisors. Let $w \neq 0$ be an element of $R_{a}$. The subgroup $w R$ is contained in $R_{a}$ since $a(w x)=(a w) x=0 x=0$. Hence the order of $w R$ is at most $n+1$. Consider the map $f: R \rightarrow w R$ defined by $(x) f=w x$ for each $x \in R$. It easily follows that $f$ is a homomorphism, that the kernel of $f$ is $R_{w}$, and that $f$ is an onto map. Thus, using the fundamental homomorphism theorem in group theory, it follows that $R / R_{w} \cong w R$. Since the order of $w R$ is the order of $R / R_{w}$, the order of $R$ is the product of the order of $w R$ and the order of $R_{w}$, which is less than or equal $(n+1)^{2}$. 
If in Theorem 2.1 right is changed to left, the conclusion does not follow. This is illustrated by

EXAMPLe 2.2. Let $(G,+)$ be an infinite group. Let $H$ be a finite subset of $G$ which contains 0 and has nonzero elements. Define $h g=0$ for each $h \in H, g \in G$ and define $x g=g$ for each $x \in G-H, g \in G$. Then $(G,+, \cdot)$ is a near-ring [7]. Each element in $H$ is a left zero divisor and $H$ is finite; but $G$ is not finite.

However, the conclusion may still be obtained if one of the left zero divisors is right distributive. This is shown in

THEOREM 2.3. Let $R$ be a near-ring with $n+1$ left zero divisors, at least one of which is right distributive. Then $R$ is finite and does not contain more than $(n+1)^{2}$ elements.

Proof. For each $x \in R$, define $L_{x}=\{y \in R \mid y x=0\}$. Note that $L_{x}$ is a subgroup if $x$ is right distributive. Let $w$ be a right distributive element that is a left zero divisor. Then there is $z \neq 0$ in $R$ such that $w z=0$. Since there is only a finite number of left zero divisors, it is seen that the order of $L_{z}$ is less than or equal to $n+1$. Since $w$ is right distributive, $R w=\{x w \mid x \in R\}$ is a subgroup of $R$. Furthermore, $R w \subseteq L_{z}$ since $(x w) z=x(w z)=x 0=0$. Hence the order of $R w$ is less than or equal to $n+1$. Consider the map $f: R \rightarrow R w$ defined by $(x) f=x w$. Because $w$ is right distributive, $f$ is a homomorphism from $R$ onto $R w$. Thus $R / \operatorname{Ker} f \cong R w$. But $\operatorname{Ker} f=R_{w}$. Since the order of $L_{w}$ is less than or equal to $n+1$, it follows that the order of $\operatorname{Ker} f$ is less than or equal $n+1$. Consequently the order of $R$ is less than or equal to $(n+1)^{2}$. This completes the proof.

\section{Intẹgral elements}

In this section a result of N. Ganesan [4] is generalized.

Defintion 3.1. Let $R$ be a near-ring. An element $x \neq 0$ in $R$ is said to be an integral element if $x$ is not a zero divisor.

Ganesan showed that the integral elements of a finite ring $R$ determine a multiplicative group whose identity is also the identity element for $R$. This result cannot be extended to arbitrary near-rings (see Example 3.3 below) but can be extended to distributively generated near-rings.

THEOREM 3.2. Let $R$ be a distributively generated near-ring with a finite number of right zero divisors and at least one integral element. Then the set of integral elements of $R$ is a multiplicative group whose identity is also the identity element for $R$.

PROof. According to Theorem 2.1, $R$ is finite. If $x$ is an integral element, then $x R=R$ and there is $e \in R$ such that $x e=x$. But $x(e x-x)=0$ and this implies that $e x=x$. Hence $e$ is an identity for $x$. For each $y \in R, x(e y-y)=0$ and this implies $e y=y$. Since $R$ is a distributively generated near-ring, $x=x_{1}+x_{2}+\cdots$ 
$+x_{n}$ where $x_{i}$ is either a right or anti-right distributive element. Thus

$$
\begin{aligned}
(y e-y) x & =(y e-y) x_{1}+(y e-y) x_{2}+\cdots+(y e-y) x_{n} \\
& = \pm\left(y e x_{1}-y x_{1}\right) \pm\left(y e x_{2}-y x_{2}\right) \pm \cdots \pm\left(y e x_{n}-y x_{n}\right) \\
& = \pm\left(y x_{1}-y x_{1}\right) \pm\left(y x_{2}-y x_{2}\right) \pm \cdots \pm\left(y x_{n}-y x_{n}\right) \\
& =0,
\end{aligned}
$$

with + chosen if $x_{i}$ is right distributive and - chosen if $x_{i}$ is anti-right distributive. The fact that $x$ is not a zero divisor implies that $y e=y$. Thus $e$ is an identity element for $R$.

It remains to be shown that the set $N$ of integral elements forms a multiplicative group and $e$ is the identity. Suppose $z, w \in N$. If there is $y \in R$ such that $(z w) y$ $=0$, then either $z$ or $w$ is a zero divisor. Thus $N$ is closed under multiplication. Since $e$ is an identity for $R$ and $e \in N$, it follows that $e$ is the identity for $N$. Now suppose $w \in N$. Since $R$ is finite, $w R=R$ and there is a $z \in R$ such that $w z=e$. Since $w(z w-e)=0$, it follows that $z$ is the multiplicative inverse of $w$. Suppose there is $x \neq 0$ in $R$ such that $z x=0$. Then $x=w z x=w 0=0$, which is a contradiction. Thus $N$ is a multiplicative group and the theorem is proved.

If a near-ring is not d.g. then the integral elements may not form a multiplicative group. This is shown by

EXAMPLE 3.3. In the near-ring given as \#10 in [2, Section 2.1] the elements 1 and 3 are the integral elements, but they do not determine a multiplicative group.

\section{References}

[1] J. C. Beidleman, On near-rings and near-ring modules, Doctoral dissertation, The Pennsylvania State University, 1964.

[2] J. R. Clay, 'The near-rings on groups of low order', Math. Z. 104 (1968), 364-371.

[3] A. Fröhlich, 'Distributively generated near-rings', Proc. London Math. Soc. (3) 8 (1958), 76-108.

[4] N. Ganesan, 'Properties of rings with a finite number of zero divisors', Math. Ann. 157 (1964) $215-218$.

[5] I. N. Herstein, Topics in algebra (Blaisdell, New York, 1964).

[6] K. Koh, 'On properties of rings with a finite number of zero divisors', Math. Ann. 171 (1967), $79-80$.

[7] J. J. Malone, 'Near-rings with trivial multiplications', Amer. Math. Monthly 74 (1967), $1111-1112$.

[8] B. H. Neumann, 'On the commutativity of addition', J. London Math. Soc. 15 (1940), 203208.

[9] H. Wefelscheid, 'Vervollstândigung topologischer Fastkörper', Math. Z. 99 (1967), 279298.

[10] H. Zassenhaus, 'Uber endliche Fastkörper', Abh. Math. Sem. Univ. Hamburg 11 (1935), $187-220$.

Department of Mathematics, Texas A \& M University

College Station, Texas 77843, United States of America 